\title{
Performance evaluation of industrial ethernet protocols for real-time fault detection based adaptive observer in networked control systems with network communication constraints
}

\author{
Samba Aimé Hervé ${ }^{1}$, Yeremou Tamtsia Aurelien ${ }^{2}$, Nneme Nneme Leandre ${ }^{3}$ \\ ${ }^{1,2}$ National Advanced School of Engineering , University of Douala, Douala, Cameroon \\ ${ }^{3}$ Advanced Teacher is Training College for Technical Education, University of Douala, Douala, Cameroon
}

\section{Article Info}

Article history:

Received Mar 20, 2020

Revised May 16, 2021

Accepted Jul 23, 2021

Keywords:

Adaptive sliding mode observer

Fault detection

Industrial ethernet

Packet losses

Networked control systems

\section{Corresponding Author:}

Samba Aimé Hervé

National Advanced School of Engineering

University of Douala, Douala, Cameroon

Email: aimehervesamba@yahoo.fr

\begin{abstract}
In this paper, the performance evaluation of industrial ethernet (EtherNet/IP, EtherCAT and PROFINET IRT) networks has been studied for choosing the right protocol in real-time fault detection based adaptive sliding mode observer in networked control systems (NCSs) under time network-induced delays, stochastic packet losses, access constraints and bounded disturbances. An adaptive sliding-mode observer based fault detection is presented. The dynamic hydroelectric power plant model is used to verify the effectiveness of the proposed method based on TrueTime and Matlab/ Simulink, corroborated our predictions that an ethernet for control automation technology (EtherCAT) protocol would be more appropriate to reduce the false alarm rate and increasing the efficiency of the remote control of industrial hydroelectric power plant.
\end{abstract}

This is an open access article under the CC BY-SA license.

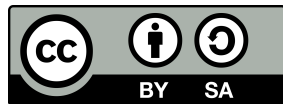

\section{INTRODUCTION}

The concept of networked control systems (NCSs) have been mentioned by many scientists in their works as early as the end of the twentieth century. Because the low maintenance cost and convenient installation, NCSs have aroused widely concerned. Due to the introduction of shared network, new constraints occur when the plant outputs and control inputs are transmitted through communication networks: quantization errors in the signals transmitted through the network, packet dropouts, network-induced delay, access constraints and power consumption mainly in wireless networked control systems [1]-[5], thus increasing the complexity of the system. These factors will affect the reliability of the system, and cause the system performance decline. In order to improve the reliability and security of the NCSs, fault diagnosis has been widely used in engineering systems such as aero engines, dynamic vehicle systems, chemical processes, and power systems [6]-[8]. Fault detection (FD) has received widespread attention as one of the most considerable parts of fault diagnosis. Failure is the phenomenon that the state of the system deviates from the normal working range due to saturation, stuck or degradation of actuators, sensors and other components, which has a negative influence on the system performance. As a result, it is very significant to detect the system faults as soon as possible to ensure the safety of systems. There are many researching results on FD for kinds of systems with various methods 
[9]-[12]. Pan and Yang [11], $H_{\infty}$ FD filter is proposed for NCS with large transfer delays. An adaptive Kalman filter for NCSs is proposed in [12] to minimize the effects of delay on the residual signal. The fault detection system for NCSs with packet losses was been designed by modelling the NCSs as a Markov jumping linear system [13], [14]. For research work of FD problem of NCSs, construct appropriate filters and state observers as residual generators to generate residual signals [15]-[17]. The problem of FD of a NCSs under communication constraints limited is considered [18]. The practical guideline for selecting the right protocol in industrial networked control systems (NCSs) is provided in [12], [19]. The evaluated the performance of MPOLSR protocol and MDART protocol using NS-2 based on the success delivery rate and packet loss bas been studied in [20]. Inspired by the above discussions, the main goal of this paper is to devoted to FD of NCS subject to both random communication delays, stochastic packet losses, limited communication and access constraints. The main contributions of this work can be highlighted as follows:

- The fault detection problem is extended for a class of networked control systems (NCSs) with random packet losses, time-varying delays and limited communication to reflect more realistic environment,

- Adaptive Sliding mode observer approach is utilized to deal with the fault detection,

- Residuals generator is designed, reducing the false alarm rate. Then, the residual signals are evaluated and compared with a threshold to detect the faults occurrences.

- Application to a Hydro-turbine governing system [21], [22] shows that the proposed method achieves better fault detection.

- True-Time toolbox is used to reflect a more realistic numerical network communication and validity of the proposed design method.

- The control performance of the proposed method is evaluated for several industrial protocols: EtherNet/IP protocol, PROFINET IRT protocol and EtherCAT protocol of the standard IEEE 802.3, the right protocol for a NCS is provided.

The remainder of this paper is organized as follows: section 2 introduces the problem statement and preliminaries, our step of adaptive observer synthesis is given in section 3. The simulation results based on True-Time toolbox and Matlab/Simulink will be given in section 4 to verify the efficiency of proposed method. Finally, a conclusion is provided, including some perspectives of this work.

\section{PROBLEM FORMULATION AND PRELIMINARIES}

In this paper, the discrete linear system with output delay is structured as Figure 1; the state-space model of the linear plant dynamics (1).

$$
\begin{aligned}
& x(k+1)=A x(k)+\mathfrak{A}_{\tau_{k}} x\left(k-\tau_{k}\right)+B u(k)+\Gamma d(k)+\mathcal{F} \Upsilon(k) \\
& y(k)=C x(k)
\end{aligned}
$$

Where $x(k) \in \mathbb{R}^{n}$ denotes the state vector, $x\left(k-\tau_{k}\right) \in \mathbb{R}^{n}$ denotes the state delay vector, $u(k) \in \mathbb{R}^{m}$ denotes the control input vector $\Upsilon(k) \in \mathbb{R}^{q}$ is the fault vector, $y(k) \in \mathbb{R}^{p}$ denotes the measured output vector and $d(k) \in \mathbb{R}^{m}$ the noise vector, $A, \mathfrak{A}_{\tau_{k}}, B, C$ and $\mathcal{F}$ are matrices of appropriate dimensions.

Assumption 1 [13]: It is supposed that random packet losses exists in output channel. It is modelled in the system as Bernoulli process. We define $\tilde{y}(k)$ the output of the system (with internal noise) and $y(k)$ the data used with a probability $\bar{\beta}\left(\Im_{r}\left\{\beta_{k}=1\right\}=\bar{\beta}\right)$. If the data is not available, we will use the preceding data $y(k-1)$ with probability $1-\bar{\beta}\left(\Im_{r}\left\{\beta_{k}=0\right\}=1-\bar{\beta}\right)$.

The following equations describe this phenomenon.

$$
y(k)=\bar{\beta} \tilde{y}(k)+(1-\bar{\beta}) y(k-1)
$$

Where $\beta_{k} \in\{0,1\}$ obeys the Bernoulli distribution.

Assumption 2 [23]: In this paper we will consider that the band-width of the communication network connecting the sensors and the fault detection module which generates a residue is limited capacity, $\varpi_{\varsigma}$ sensors among $p$ can reach these channels to communicate with the residues generator while the others remain on standby. Similarly, $\varpi_{\varrho}$ from $p$ actuators receive their command from controller at each sampling period. 
where

$$
\begin{aligned}
& 1 \leq \varpi_{\varrho} \leq m \\
& 1 \leq \varpi_{\varsigma} \leq p
\end{aligned}
$$

Taking the phenomenon of sensor and actuator saturation, the functions of saturation $\varsigma(\cdot): \mathbb{R}^{m} \rightarrow \mathbb{R}^{m}$ and $\varrho(\cdot): \mathbb{R}^{m} \rightarrow \mathbb{R}^{m}$ are defined as (4).

$$
\begin{aligned}
& \varsigma(k)=\left[\varsigma_{1}^{T}(k), \varsigma_{2}^{T}(k), \cdots, \varsigma_{m}^{T}(k)\right]^{T} \\
& \varrho(k)=\left[\varrho_{1}^{T}(k), \varrho_{2}^{T}(k), \cdots, \varrho_{m}^{T}(k)\right]^{T}
\end{aligned}
$$

If we denote by $\bar{y}(k)$ denotes measurement vector available to controller and fault detection module and $\bar{u}(k)$ denote the control signals generated by controller at discrete time $k$. Based on the above communication sequence, We get the relations in (5).

$$
\begin{gathered}
\bar{y}(k)=M_{\varsigma}(k) \cdot y(k), M_{\varsigma} \triangleq \operatorname{diag}\left(\varsigma_{i}(k)\right) \\
u(k)=M_{\varrho}(k) \cdot \bar{u}(k), M_{\varrho} \triangleq \operatorname{diag}\left(\varrho_{i}(k)\right)
\end{gathered}
$$

From (1), (2), (3), (4) and (5), the dynamics of the networked control system via a shared communication medium can be described as (6).

$$
\begin{aligned}
& x(k+1)=A x(k)+\mathfrak{A}_{\tau_{k}} x\left(k-\tau_{k}\right)+B M_{\varrho}(t) \bar{u}(k)+\Gamma d(k)+\mathcal{F} \Upsilon(k), \\
& y(k)=\bar{\beta} \tilde{y}(k)+(1-\bar{\beta}) y(k-1) \\
& \bar{y}(k)=M_{\varsigma}(t) C x(k)
\end{aligned}
$$

Lemma 1 [24] (Schur complement). Given constant matrices of appropriate dimensions $\mathfrak{B}_{11}, \mathfrak{B}_{12}$ and $\mathfrak{B}_{22} \in$ $\mathbb{R}^{n \times n}$, where $\mathfrak{B}_{11}=\mathfrak{B}_{11}^{T}, \mathfrak{B}_{22}=\mathfrak{B}_{22}^{T}$, then $\mathfrak{B}_{12}>0, \mathfrak{B}_{11}-\mathfrak{B}_{12} \mathfrak{B}_{22}^{-1} \mathfrak{B}_{12}^{T}>0$ if and only if

$$
\left[\begin{array}{cc}
\mathfrak{B}_{11} & \mathfrak{B}_{22} \\
\mathfrak{B}_{12}^{T} & -\mathfrak{B}_{22}
\end{array}\right]>0
$$

Lemma 2 [25] Given matrices of appropriate dimensions $\Xi_{11}=\Xi_{11}^{T}, \Xi_{12}$ and $\Xi_{22} \cdot \mathfrak{F}$ a function which satisfies $\mathfrak{F}=\mathfrak{F}^{T} \leq I$, where is an identity matrix. Then the inequality $\Xi_{11}+\Xi_{12} \mathfrak{F} \Xi_{22}+\Xi_{22}^{T} \mathfrak{F}^{T} \Xi_{12}^{T}<0$. Is not true that, if and only if there exists a scalar $\mathfrak{Z}$ such as the inequality is checked: $\Xi_{11}+\mathfrak{Z} \Xi_{12} \Xi_{12}^{T}+\mathfrak{Z}^{-1} \Xi_{22} \Xi_{22}^{T}<0$. or equivalently

$$
\left[\begin{array}{ccc}
\Xi_{11} & \mathfrak{Z}_{12} & \Xi_{22}^{T} \\
* & -\mathfrak{Z} \Xi_{12} & 0 \\
* & * & -\mathfrak{Z}_{12}
\end{array}\right]<0 .
$$

where the symbols $(*)$ denote the symmetric terms.

The remote FD dynamic behaviour of NCSs is illustrated in Figure 1. Notably, $y(k)$ denotes the actual output signal and $\tilde{y}(k)$ denotes the output signal used by controller, $u(k)$ is the control signal produced by controller and $\bar{u}(k)$ is the actual control input. In this structure the fault information is not affected by the communication delay between the sensor and the node of FD unit and communication delay between controller and the node of FD unit. 


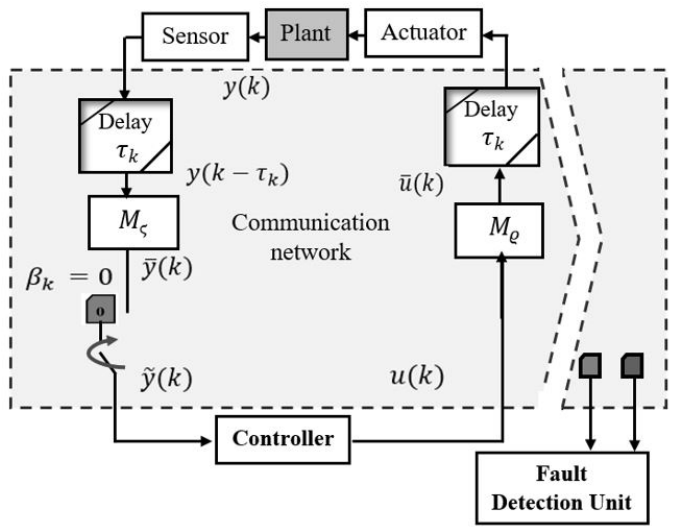

Figure 1. The proposed block diagram of remote FD in networked control systems with time delays, packet losses and access constraints

\section{RESIDUAL DYNAMIC SYSTEMS}

In this section, we aim to design an fault observer based on discrete-time adaptive sliding mode for considered networked system with packet losses, communication delays and access constraints. The structure of the adaptive fault observer proposed is given by (7).

$$
\begin{aligned}
& \hat{x}(k+1)=A \hat{x}(k)+\mathfrak{A}_{\tau_{k}} \hat{x}\left(k-\tau_{k}\right)+B M_{\varrho}(k) \bar{u}(k)+L[y(k)-\hat{y}(k)] \\
& \hat{\Upsilon}(k+1)=\Lambda_{1} \hat{\Upsilon}(k)+\Lambda_{2}(y(k)-\hat{y}(k)) \\
& \hat{y}(k)=(1-\bar{\beta}) M_{\varsigma}(k) C \hat{x}(k)
\end{aligned}
$$

Where $\hat{x} \in \mathbb{R}^{n}$ and $\hat{y}(k) \in \mathbb{R}^{p}$ denote the state estimation for the fault observer and estimation of the measurement output respectively; $L \in \mathbb{R}^{n \times p}$ is the the observer gain matrix and $\hat{\Upsilon}(k)$ is the estimation of the fault vector. This adaptive sliding mode observer allows to generate a residue which will be analyzed to detect the faults. It is significant to note that in the context considered in this paper, $\Lambda_{1}, \Lambda_{2}$ are the fault detection parameters.

Let $\varepsilon_{x}(k)=x(k)-\hat{x}(k)$ and $\varepsilon_{y}(k)=y(k)-\hat{y}(k)$.

According to (1) and (7), we get the estimaror dynamics by (8).

$$
\begin{gathered}
\varepsilon_{x}(k+1)=\left(A-(1-\bar{\beta}) M_{\varsigma} L C\right) \varepsilon(k)+(1-\bar{\beta}) L C x(k)+\Gamma d(k) \\
+\mathcal{F} \check{\Upsilon}(k)+\mathfrak{A}_{\tau_{k}} \varepsilon_{x}\left(k-\tau_{k}\right) \\
\varepsilon_{y}(k)=(1-\bar{\beta}) M_{\varsigma}(k) C \varepsilon_{x}(k)
\end{gathered}
$$

where $\breve{\Upsilon}(k)=\Upsilon(k)-\hat{\Upsilon}(k)$

For the fault observer, the logic of fault detection considered in this work is given by (10).

$$
\begin{array}{ll}
\|\mathfrak{J}(k)\|>\mathfrak{J}_{t h} & \text { Alarm, fault is detected } \\
\|\mathfrak{J}(k)\| \leq \mathfrak{J}_{t h} & \text { No alarm, fault is no detected }
\end{array}
$$

Where $\mathfrak{J}(k)$ is the residual evaluation function of the residual generator and $\mathfrak{J}_{\mathfrak{t h}}$ is the threshold are selected as (11).

$$
\begin{aligned}
& \mathfrak{J}(k)=\left[\sum_{k=1}^{n} r(k)^{T} r(k)\right]^{\frac{1}{2}} \\
& \mathfrak{J}_{t h}=\sup _{d(k) \in I_{2} \Upsilon(k)=0} \mathbb{E}\{\mathfrak{J}(k)\}
\end{aligned}
$$

where $n$ is the length of the evaluation window and $r(k))=\varepsilon_{y}(k)$ denotes the residual signal of the system. 


\section{STABILITY ANALYSIS}

Theorem 1. Consider the system (7) with the given scalar $\gamma>0$, if there exist matrices $\mathbb{P}_{1}>0, \Lambda_{1}$, $\Lambda_{2}>0$ and $L$, such that the following matrix inequality holds.

$$
\mathcal{H}=\left[\begin{array}{ccc}
\Sigma_{11} & \gamma \Sigma_{12} & \Sigma_{22}^{T} \\
* & -\gamma \Sigma_{12} & 0 \\
* & * & -\gamma \Sigma_{12}
\end{array}\right]<0
$$

where

$$
\begin{gathered}
\Sigma_{11}=\left[\begin{array}{cc}
-\mathbb{P}_{1}+\gamma \mathbb{I} & 0 \\
0 & -\mathbb{I}+\gamma \mathbb{I}
\end{array}\right], \\
\Sigma_{22}=\left[\begin{array}{cc}
-\mathbb{P}_{1} & 0 \\
0 & -\mathbb{I}
\end{array}\right] \\
\Sigma_{21}=\left[\begin{array}{cc}
\tilde{\Sigma}_{11} & \tilde{\Sigma}_{12} \\
\tilde{\Sigma}_{21} & \tilde{\Sigma}_{22}
\end{array}\right] \\
\tilde{\Sigma}_{12}=\left(\Lambda_{2}(1-\bar{\beta}) M_{\varsigma} C\right)^{T} \\
\tilde{\Sigma}_{21}=\mathcal{F}^{T} \mathbb{P}_{1} \\
\tilde{\Sigma}_{22}=\Lambda_{1}^{T}
\end{gathered}
$$

Then the residual dynamic system (7) is asymptotically stable.

Proof 1. Choose a Lyapunov functional candidate as (13).

$$
\mathbb{V}(k)=\varepsilon_{x}^{T}(k) \mathbb{P}_{1} \varepsilon_{x}(k)+\breve{\Upsilon}^{T}(k) \breve{\Upsilon}(k)
$$

Then, it can be obtained as (14).

$$
\begin{aligned}
\mathbb{E}\{\mathbb{V}(k+1)-\mathbb{V}(k)\}= & \mathbb{E}\left\{\varepsilon_{x}(k)^{T}\left(A-(1-\bar{\beta}) M_{\varsigma} L C\right)^{T} \mathbb{P}_{1}\left(A-(1-\bar{\beta}) M_{\varsigma} L C\right) \varepsilon_{x}(k)\right. \\
& +2 \varepsilon_{x}(k)\left(A-(1-\bar{\beta}) M_{\varsigma} L C\right)^{T} \mathbb{P}_{1} \mathcal{F} \breve{\Upsilon}(k)+\breve{\Upsilon}^{T}(k) \mathcal{F}^{T} \mathbb{P}_{1} \mathcal{F} \breve{\Upsilon}(k) \\
& +\breve{\Upsilon}^{T}(k+1) \breve{\Upsilon}(k+1) \varepsilon_{x}\left(k-\tau_{k}\right)^{T} \mathfrak{A}_{\tau_{k}}^{T} \mathbb{P}_{1} \mathfrak{A}_{\tau_{k}} \varepsilon_{x}\left(k-\tau_{k}\right) \\
& +2 e_{x}(k)^{T}\left(A-(1-\bar{\beta}) M_{\varsigma} L C\right)^{T} \mathbb{P}_{1} \mathfrak{A}_{\tau_{k}} \times e_{x}\left(k-\tau_{k}\right) \\
& +2 \varepsilon_{x}^{T}(k)\left(A-(1-\bar{\beta}) M_{\varsigma} L C\right)^{T}\left(A-(1-\bar{\beta}) M_{\varsigma} L C\right)^{T} \mathbb{P}_{1} \\
& \times \Gamma d(k)+2 e\left(k-\tau_{k}\right)^{T} \mathfrak{A}_{\tau_{k}} \mathbb{P}_{1} \Gamma d(k)+[\Gamma d(k)]^{T} \mathbb{P}_{1}[\Gamma d(k)] \\
& +\Gamma d(k)-\varepsilon_{x}(k)^{T} \mathbb{P}_{1} \varepsilon_{x}(k)-\breve{\Upsilon}^{T}(k) \breve{\Upsilon}(k)
\end{aligned}
$$

where

$$
\begin{aligned}
& \mathbb{E}\{\breve{\Upsilon}(k+1)\}=-\Lambda_{1} \tilde{\Upsilon}(k)-\Lambda_{2} \varepsilon_{x}(k)+\Phi(k) \\
& \Phi(k)=\Lambda_{1} \Upsilon(k)+\Upsilon(k+1)
\end{aligned}
$$

Taking $\|d(k)\| \leq \bar{d}$, where $\bar{d}$ is know positive constants, it is derived that

$$
\begin{gathered}
{[\Gamma d(k)]^{T} \mathbb{P}_{1}[\Gamma d(k)] \leq\|\Gamma d(k)\|^{2}\left\|\mathbb{P}_{1}\right\| \leq[\|\Gamma\| \bar{d}]^{2}\left\|\mathbb{P}_{1}\right\|} \\
{\left[A-(1-\bar{\beta}) M_{\varsigma} L C\right]^{T} \mathbb{P}_{1}[\Gamma d(k)] \leq\left\|\left[A-(1-\bar{\beta}) M_{\varsigma} L C\right]^{T} \mathbb{P}_{1}\right\|\|\Gamma d(k)\|} \\
2 \varepsilon_{x}\left(k-\tau_{k}\right)^{T} \mathfrak{A}_{\tau_{k}}^{T} \mathbb{P}_{1}[\Gamma d(k)] \leq 2\left\|\varepsilon_{x}\left(k-\tau_{k}\right)^{T} \mathfrak{A}_{\tau_{k}}^{T}\right\|\left\|\mathbb{P}_{1}\right\|[\|\Gamma\| \bar{d}]
\end{gathered}
$$


According to (15), (16), (17) and (18). It can be further obtained (19).

$$
\begin{aligned}
\mathbb{E}\{\mathbb{V}(k+1)-\mathbb{V}(k)\} \leq & \varepsilon_{x}(k)^{T}\left\{\left(A-(1-\bar{\beta}) M_{\varsigma} L C\right)^{T} \mathbb{P}_{1}\left(A-(1-\bar{\beta}) M_{\varsigma} L C\right)-\mathbb{P}_{1}\right\} \\
& \times \varepsilon_{x}(k)+2 \varepsilon_{x}(k)\left(A-(1-\bar{\beta}) M_{\varsigma} L C\right)^{T} \mathbb{P}_{1} \mathcal{F} \breve{\Upsilon}(k)+\breve{\Upsilon}^{T}(k) \\
& \times\left(\mathcal{F}^{T} \mathbb{P}_{1} \Upsilon(k)-\mathbb{I}\right) \breve{\Upsilon}(k)+2\left\|\left(A-(1-\bar{\beta}) M_{\varsigma} L C\right)^{T} \mathbb{P}_{1}\right\| \\
& \times\left\|\varepsilon_{x}(k)^{T}\right\|\|\Gamma\| \bar{d}\|+2\| \varepsilon_{x}\left(k-\tau_{k}\right)^{T} \mathfrak{A}_{\tau_{k}}^{T}\|\| \mathbb{P}_{1} \| \\
& \times[\|\Gamma\| \bar{d}]+\varepsilon_{x}(k)^{T}\left(A-(1-\bar{\beta}) M_{\varsigma} L C\right)^{T} \mathbb{P}_{1} \\
& \times\left(A-(1-\bar{\beta}) M_{\varsigma} L C\right) \varepsilon_{x}(k)+2 \lambda^{2} \varepsilon_{x}(k)^{T} \mathbb{P}_{1} \varepsilon_{x}(k) \\
& +\mathbb{M}^{T} \Theta^{T}+\Phi(k)\left(\mathbb{M}^{T} \Theta+\Phi(k)\right)\left\|\mathbb{P}_{1}\right\|+[\|\Gamma\| \bar{d}]^{2}
\end{aligned}
$$

where,

$$
\begin{aligned}
& \mathbb{M}^{T}=\left[\begin{array}{cc}
\varepsilon_{x}(k)^{T} & \breve{\Upsilon}^{T}(k)
\end{array}\right] \\
& \Theta^{T}=\left[\begin{array}{c}
-\left(\Lambda_{2}(1-\bar{\beta}) M_{\varsigma} C^{T}\right) \\
-\Lambda_{1}
\end{array}\right]
\end{aligned}
$$

According to the Lemma 2 and (19), it easy to obtain (20).

$$
\mathbb{E}\{\mathbb{V}(k+1)-\mathbb{V}(k)\} \leq \mathbb{M}^{T} \mathcal{H} \mathbb{M}+2 \mathbb{M}^{T} \Theta^{T} \Phi(k)+\Phi(k)^{T} \Phi(k)
$$

For the matrix $\mathcal{H}$, it can be obtained by Lemma 1 :

$$
\mathcal{H}=\left[\begin{array}{ccc}
\Sigma_{11} & \gamma \Sigma_{12} & \Sigma_{22}^{T} \\
* & -\gamma \Sigma_{12} & 0 \\
* & * & -\gamma \Sigma_{12}
\end{array}\right]<0
$$

where

$$
\begin{aligned}
& \text { where } {\left[\begin{array}{cc}
-\mathbb{P}_{1}+\gamma \mathbb{I} & 0 \\
0 & -\mathbb{I}+\gamma \mathbb{I}
\end{array}\right], } \\
& \Sigma_{11}=\left[\begin{array}{cc}
-\mathbb{P}_{1} & 0 \\
0 & -\mathbb{I}
\end{array}\right], \\
& \Sigma_{22}=\left[\begin{array}{cc}
\tilde{\Sigma}_{11} & \tilde{\Sigma}_{12} \\
\tilde{\Sigma}_{21} & \tilde{\Sigma}_{22}
\end{array}\right], \\
& \Sigma_{21}=\left(A-(1-\bar{\beta}) M_{\varsigma} L C\right)^{T} \mathbb{P}_{1}, \\
& \tilde{\Sigma}_{11}=(A) \tilde{\Sigma}_{12}=\left(\Lambda_{2}(1-\bar{\beta}) M_{\varsigma} C\right)^{T}, \\
& \tilde{\Sigma}_{21}=\mathcal{F}^{T} \mathbb{P}_{1}, \\
& \tilde{\Sigma}_{22}=\Lambda_{1}^{T},
\end{aligned}
$$

The proof is completed.

Theorem 2. Consider the dynamic system (8). If there exist matrices $L \in \mathbb{R}^{n \times m}$ and $\eta^{2}<1$ satisfying the condition.

$$
\left[\begin{array}{cc}
\bar{\Omega}_{11} & \bar{\Omega}_{12} \\
* & \bar{\Omega}_{22}
\end{array}\right]<0
$$

\section{Where}

$$
\begin{aligned}
& \bar{\Omega}_{11}=-\eta^{2}\left((1-\bar{\beta}) M_{\varsigma}(k) C\right)^{T}\left((1-\bar{\beta}) M_{\varsigma}(k) C\right), \\
& \bar{\Omega}_{12}=2\left(A-(1-\bar{\beta}) M_{\varsigma} L C\right)^{T}, \\
& \bar{\Omega}_{22}=-\frac{1}{2}\left((1-\bar{\beta}) M_{\varsigma}(k) C\right)^{T}\left((1-\bar{\beta}) M_{\varsigma}(k) C\right),
\end{aligned}
$$

then system motion gets into the sliding surface in finite time. 

satisfies.

Proof 2. According to [22], the system motion gets into the sliding surface in finite time, then $\mathfrak{s}(k)$

$$
\|\mathfrak{s}(k+1)\| \leq\|\mathfrak{s}(k)\|
$$

the condition (23) can then be reformulated in (24).

$$
\mathfrak{s}(k+1)^{T} \mathfrak{s}(k+1)-\eta^{2} \mathfrak{s}(k)^{T} \mathfrak{s}(k)=\varepsilon_{y}^{T}(k+1) \varepsilon_{y}(k+1)-\eta^{2} \varepsilon_{y}^{T}(k) \varepsilon_{y}(k)
$$

According to (8), then we have

$$
\begin{aligned}
\mathfrak{s}(k+1)^{T} \mathfrak{s}(k+1)-\eta^{2} \mathfrak{s}(k)^{T} \mathfrak{s}(k)= & \varepsilon_{x}^{T}(k+1)\left((1-\bar{\beta}) M_{\varsigma}(k) C\right)^{T}\left((1-\bar{\beta}) M_{\varsigma}(k) C\right) \\
& \times \varepsilon_{x}(k+1)-\eta^{2} \varepsilon_{x}^{T}(k)\left((1-\bar{\beta}) M_{\varsigma}(k) C\right)^{T} \\
& \times\left((1-\bar{\beta}) M_{\varsigma}(k) C\right) \varepsilon_{x}(k)<0
\end{aligned}
$$

where $\eta^{2}<1$, then substituting (8) into (26), we can obtain (26).

$$
\begin{aligned}
\mathfrak{s}(k+1)^{T} \mathfrak{s}(k+1)-\eta^{2} \mathfrak{s}(k)^{T} \mathfrak{s}(k)= & \varepsilon_{x}^{T}(k)\left[\left(A-(1-\bar{\beta}) M_{\varsigma} L C\right)^{T}\left((1-\bar{\beta}) M_{\varsigma}(k) C\right)^{T}\right. \\
& \times\left((1-\bar{\beta}) M_{\varsigma}(k) C\right)\left(A-(1-\bar{\beta}) M_{\varsigma} L C\right) \\
& \left.-\eta^{2}\left((1-\bar{\beta}) M_{\varsigma}(k) C\right)^{T}\left((1-\bar{\beta}) M_{\varsigma}(k) C\right)\right] \\
& \times \varepsilon_{x}^{T}(k)+\varepsilon_{x}\left(k-\tau_{k}\right)^{T} \mathfrak{A}_{\tau_{k}}^{T}\left((1-\bar{\beta}) M_{\varsigma}(k) C\right)^{T} \\
& \times\left((1-\bar{\beta}) M_{\varsigma}(k) C\right) \varepsilon_{x}\left(k-\tau_{k}\right)+[\Gamma d(k)]^{T} \\
& \times\left((1-\bar{\beta}) M_{\varsigma}(k) C\right)^{T}\left((1-\bar{\beta}) M_{\varsigma}(k) C\right) \\
& \times \Gamma d(k)+2 \varepsilon_{x}^{T}(k) \times\left(A-(1-\bar{\beta}) M_{\varsigma} L C\right)^{T} \\
& \times\left((1-\bar{\beta}) M_{\varsigma}(k) C\right)^{T}\left((1-\bar{\beta}) M_{\varsigma}(k) C\right) \mathfrak{A}_{\tau_{k}} \\
& \times \varepsilon_{x}\left(k-\tau_{k}\right)+2 \varepsilon_{x}^{T}(k)\left(A-(1-\bar{\beta}) M_{\varsigma} L C\right)^{T} \\
& \times\left((1-\bar{\beta}) M_{\varsigma}(k) C\right)^{T}\left((1-\bar{\beta}) M_{\varsigma}(k) C\right) \Gamma d(k) \\
& +2 \varepsilon_{x}^{T}(k)\left(A-(1-\bar{\beta}) M_{\varsigma} L C\right)^{T} \mathfrak{A}_{\tau_{k}}^{T} \\
& \times\left((1-\bar{\beta}) M_{\varsigma}(k) C\right)^{T}\left((1-\bar{\beta}) M_{\varsigma}(k) C\right) \Gamma d(k)
\end{aligned}
$$

Simolarly, (24) can be obtained (27).

$$
\begin{aligned}
s(k+1)^{T} s(k+1)-\eta^{2} s(k)^{T} s(k) \leq & \varepsilon_{x}^{T}(k)\left[\left(A-(1-\bar{\beta}) M_{\varsigma} L C\right)^{T}\left((1-\bar{\beta}) M_{\varsigma}(k) C\right)^{T}\right. \\
& -\eta^{2}\left((1-\bar{\beta}) M_{\varsigma}(k) C\right)^{T}\left((1-\bar{\beta}) M_{\varsigma}(k) C\right. \\
& \left.\left.\times\left(A-(1-\bar{\beta}) M_{\varsigma} L C\right)\left((1-\bar{\beta}) M_{\varsigma}(k) C\right)\right] \varepsilon_{x}(k)\right) \\
& +2 \varepsilon_{x}^{T}(k)\left(A-(1-\bar{\beta}) M_{\varsigma} L C\right)^{T}\left((1-\bar{\beta}) M_{\varsigma}(k) C\right)^{T} \\
& \times\left((1-\bar{\beta}) M_{\varsigma}(k) C\right) \mathfrak{A}_{\tau_{k}} \varepsilon_{x}\left(k-\tau_{k}\right)-2 \varepsilon_{x}^{T}(k) \\
& \times\left(A-(1-\bar{\beta}) M_{\varsigma} L C\right)^{T}\left((1-\bar{\beta}) M_{\varsigma}(k) C\right)^{T} \\
& \times\left((1-\bar{\beta}) M_{\varsigma}(k) C\right) \Lambda_{2}(k)-2 \varepsilon_{x}\left(k-\tau_{k}\right)^{T} \\
& \times\left((1-\bar{\beta}) M_{\varsigma}(k) C\right)^{T}\left((1-\bar{\beta}) M_{\varsigma}(k) C\right) \Lambda(k)
\end{aligned}
$$

One can further get (28).

$$
\begin{aligned}
\mathfrak{s}(k+1)^{T} \mathfrak{s}(k+1)-\eta^{2} \mathfrak{s}(k)^{T} \mathfrak{s}(k) \leq & \varepsilon_{x}^{T}(k)\left[2\left(A-(1-\bar{\beta}) M_{\varsigma} L C\right)^{T}\left((1-\bar{\beta}) M_{\varsigma}(k) C\right)^{T}\right. \\
& \times\left((1-\bar{\beta}) M_{\varsigma}(k) C\right)\left(A-(1-\bar{\beta}) M_{\varsigma} L C\right) \\
& \left.-\eta^{2}\left((1-\bar{\beta}) M_{\varsigma}(k) C\right)^{T}\left((1-\bar{\beta}) M_{\varsigma}(k) C\right)\right] \\
& \times \varepsilon_{x}(k)<0
\end{aligned}
$$


According to the Lemma 2, we obtain (29).

$$
\mathfrak{s}(k+1)^{T} s(k+1)-\eta^{2} \mathfrak{s}(k)^{T} \mathfrak{s}(k) \leq \varepsilon_{x}^{T}(k) \bar{\Xi} \varepsilon_{x}(k)<0
$$

Where

$$
\begin{aligned}
& \bar{\Xi}=\left[\begin{array}{cc}
\bar{\Xi}_{11} & \bar{\Xi}_{12} \\
* & \bar{\Xi}_{22}
\end{array}\right], \\
& \bar{\Xi}_{11}=-\eta^{2}\left((1-\bar{\beta}) M_{\varsigma}(k) C\right)^{T} \times\left((1-\bar{\beta}) M_{\varsigma}(k) C\right) \\
& \bar{\Xi}_{12}=2\left(A-(1-\bar{\beta}) M_{\varsigma} L C\right)^{T} \\
& \bar{\Xi}_{22}=-\frac{1}{2}\left((1-\bar{\beta}) M_{\varsigma}(k) C\right)^{T}\left((1-\bar{\beta}) M_{\varsigma}(k) C\right)
\end{aligned}
$$

This completes the proof.

\section{SIMULATION RESULTS}

In this section, we will propose a numerical example of simulation to illustrate the effectiveness of the methods presented in this work. Let us consider the model of networked control hydroelectric power plant [20]. The flowchart of the winnowing device control and communication network is represented on Figure 2.

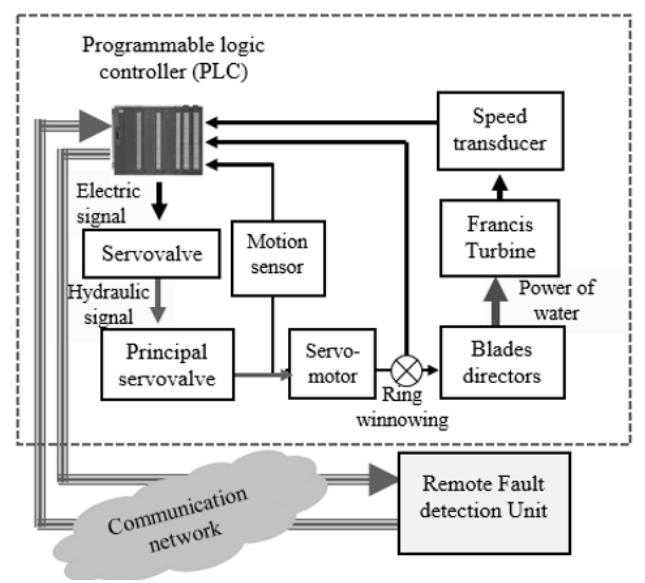

Figure 2. Schematic diagram of networked control hydroelectric power plant

This system is been used in [21], [25], for the design A Networked iterative learning fault Diagnosis algorithm for systems with sensor random packet losses, time-varying delays, limited communication and actuator failure.

The state representation of dynamic model is described as (30).

$$
\begin{aligned}
& \begin{array}{c}
x(k+1)=\left[\begin{array}{ccc}
1.1840 & -0.4046 & 0 \\
0.5000 & 0 & 0 \\
0 & 0.5000 & 0
\end{array}\right] x(k)+\left[\begin{array}{l}
1 \\
0 \\
0
\end{array}\right] u(k) \\
y(k)=\left[\begin{array}{ccc}
0.2943 & 0.3382 & 0.0001
\end{array}\right] x(k)
\end{array} \\
& \text { Define } \mathfrak{A}_{\tau_{k}}=\left[\begin{array}{ccc}
0.034 & 0 & -0.01 \\
0.031 & 0.03 & 0 \\
0.04 & 0.05 & -0.01
\end{array}\right]
\end{aligned}
$$

According to the time scale, we define the time-varying communication delays as $\tau_{i}(k)(i=0,1,2)$. 
Let the fault signal $\Upsilon(k)$ be given as (31).

$$
\Upsilon(k)=\left\{\begin{array}{cc}
0.5+0.2 \sin (k) & 122 \leq k \leq 142 \\
0, & \text { others }
\end{array}\right.
$$

And $\mathcal{F}=\left[\begin{array}{lll}1 & 1 & 1\end{array}\right]^{T}$

The communication constraints is fixed to one channel $\left(\varpi_{\varsigma}=\varpi_{\varrho}=1\right)$ so the following 3-periodic sequence can be proposed.

$$
\begin{aligned}
& \{\varsigma(0), \varsigma(1), \varsigma(2), \cdots\}=\left\{\left[\begin{array}{l}
1 \\
0 \\
0
\end{array}\right],\left[\begin{array}{l}
0 \\
1 \\
0
\end{array}\right],\left[\begin{array}{l}
0 \\
0 \\
1
\end{array}\right],\left[\begin{array}{l}
1 \\
0 \\
0
\end{array}\right], \cdots\right\} \\
& \{\varrho(0), \quad \varrho(1), \quad \varrho(2), \cdots\}=\left\{\left[\begin{array}{l}
1 \\
0 \\
0
\end{array}\right],\left[\begin{array}{l}
0 \\
1 \\
0
\end{array}\right],\left[\begin{array}{l}
0 \\
0 \\
1
\end{array}\right],\left[\begin{array}{l}
1 \\
0 \\
0
\end{array}\right], \cdots\right\}
\end{aligned}
$$

The random variable $\bar{\beta}$ satisfies the Bernoulli distribution, let $\bar{\beta}=0.65$.

Applying Theorem 1 and 2, we can obtain according to the Matlab LMI toolbox, the desired $\mathbb{P}_{1}$, the observer gain $L$, the scalar $\eta$ and parameters $\Lambda_{1}$ and $\Lambda_{1}$ as follows:

$$
\begin{aligned}
& \mathbb{P}_{1}=\left[\begin{array}{ccc}
0.231 & -0.005 & 0.002 \\
0 & 0.0012 & 0.0021 \\
0 & 0.0321 & 0.0063
\end{array}\right], \\
& L=\left[\begin{array}{c}
-0.0352 \\
0.000011 \\
-0.0142
\end{array}\right], \Lambda_{1}=0.03621, \Lambda_{2}=0.0073 \text { and } \eta=1.0173 e^{-3} .
\end{aligned}
$$

We obtain the Simulink/ true time models of networked control hydroelectric power plant Figure 3. The true-time network block simulates the access to the medium and allows the transmission and the reception of data through the network. Table 1 shows the simulation parameter for wireless network block.

Table 1. Simulation parameter for network block

\begin{tabular}{lc}
\hline Parameter & Values \\
\hline Network type & $802.15(\mathrm{LAN})$ \\
Data rate & $200 \mathrm{Mps}$ \\
Minimum frame size & $544 \mathrm{bits}$ \\
Transmit power & $200 \mathrm{dbm}$ \\
Receiver signal threshold & $-48 \mathrm{dbm}$ \\
Path loss exponent & 33.5 \\
\hline
\end{tabular}

In order to show the effectiveness of the approach proposed, the noise signal $d(k)$ is a white noise Gaussian of an amplitude of 0.025 (sample time $T_{s}=1 s$ ). The fault signal $\Upsilon(k)$ occurs between the moments $122^{\text {th }}$ at $157^{\text {th }}$ steps.

The results comprise a packets loss at the moments: steps 11 at steps 19 steps, steps 41 at steps 52 , steps 63 at steps 68 , steps 84 at steps 86 , steps 89 , steps 101 at steps 108, steps 128, steps 133 at steps 139 and steps 194 at steps 196 Figure 4.

Figure 5 show the evolution in real time of the actual speed of hydroturbine and the estimated speed by adaptive sliding mode observer with EtherNet/IP protocol, PROFINET IRT protocol and EtherCAT protocol.

The generated residue is illustrated by the Figure 6, which shows that the residual signal converges to zero without faults Figure 7 , then, changes rapidly when the faults occurred this for the three communications protocols. Figure 8 illustrate the residual evaluation function $\mathfrak{J}(k)$.

Figure 9 shows that the same faults is detected at steps 125 with switched ethernet protocol. Figure 10 shows the occurrence of fault can detect the fault at steps 124 with EtherCAT protocol. 


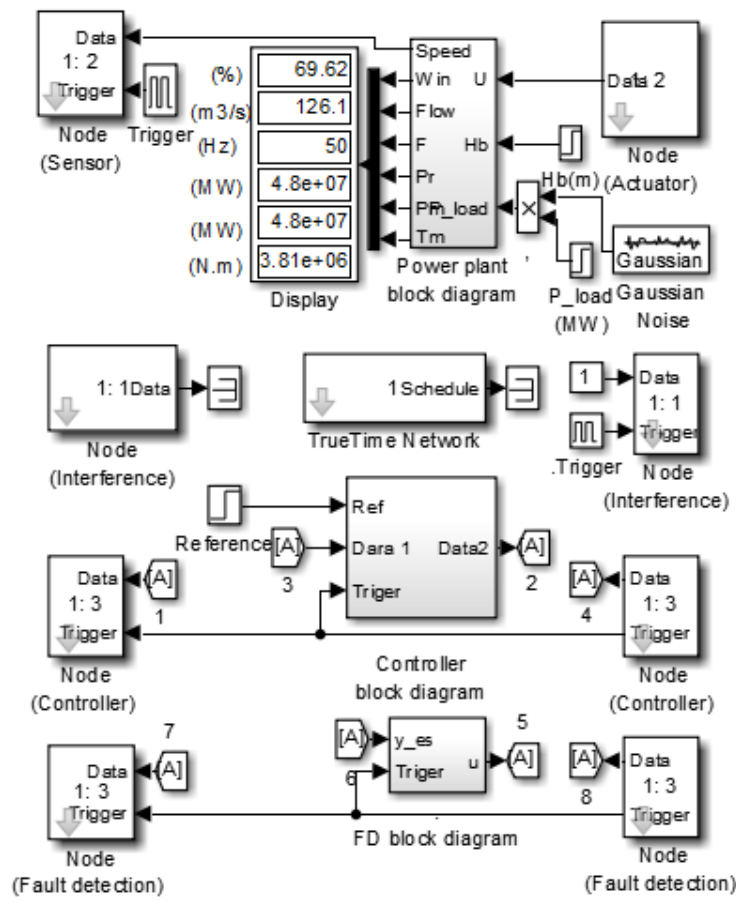

Figure 3. Simulink/true-time model of remote FD in networked control hydroelectric power plant

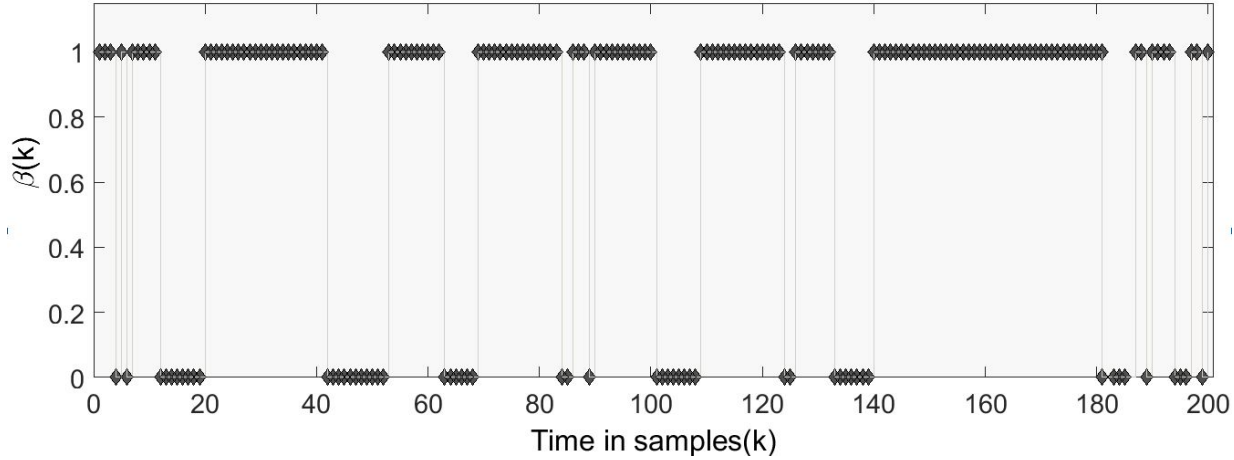

Figure 4. The distribution of packet losses "1" means packet received, "0" means packet lost

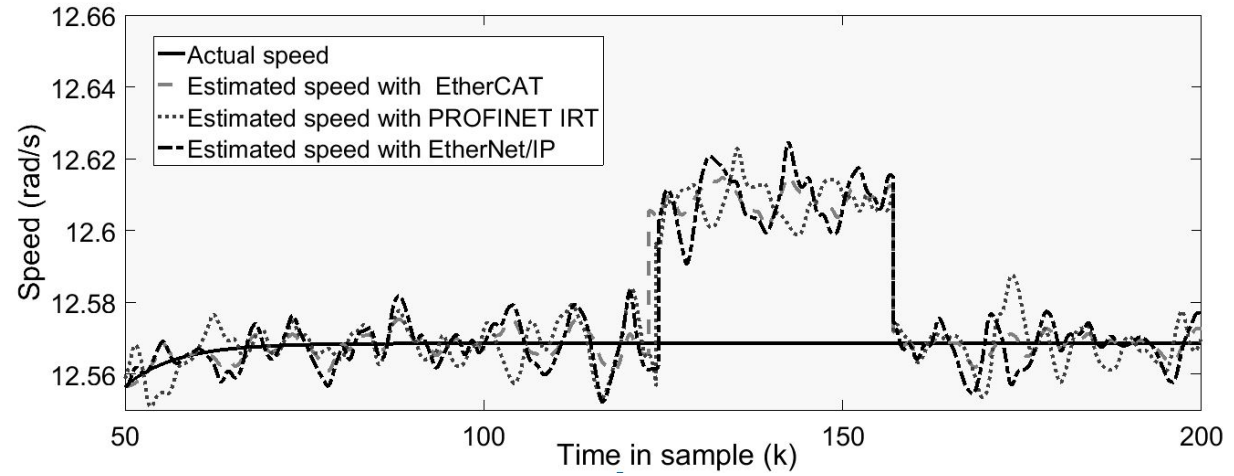

Figure 5. Actual and estimated speed of hydroturbine

Int J Rob \& Autom, Vol. 10, No. 3, September 2021 : 261 - 274 


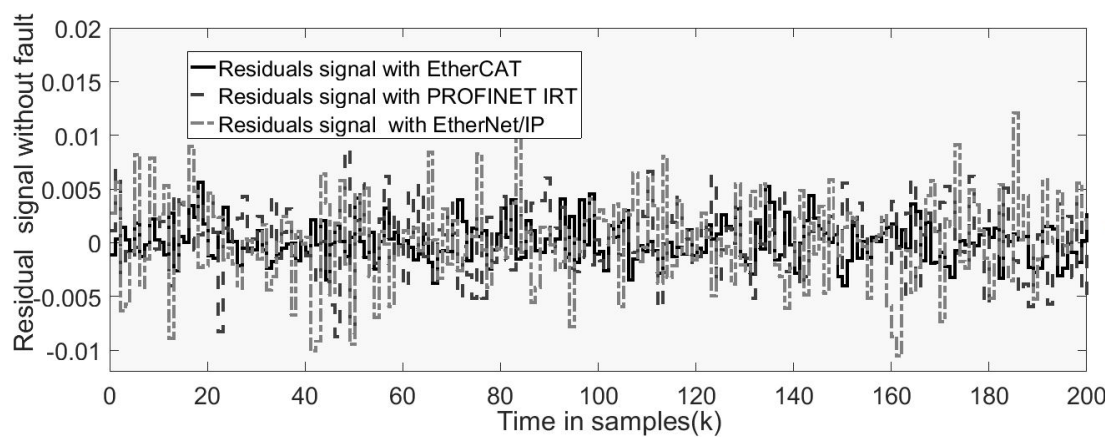

Figure 6. Residual signal r(k) with fault

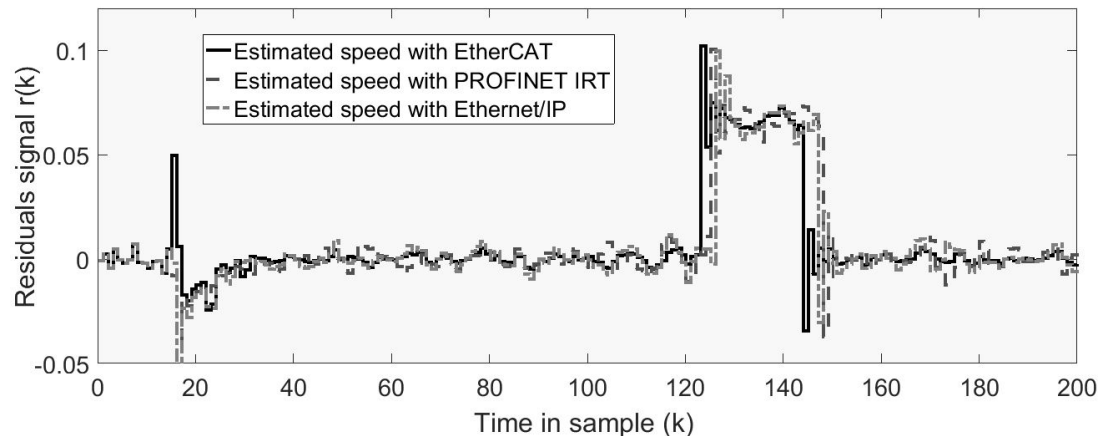

Figure 7. Residual signal r(k) without fault

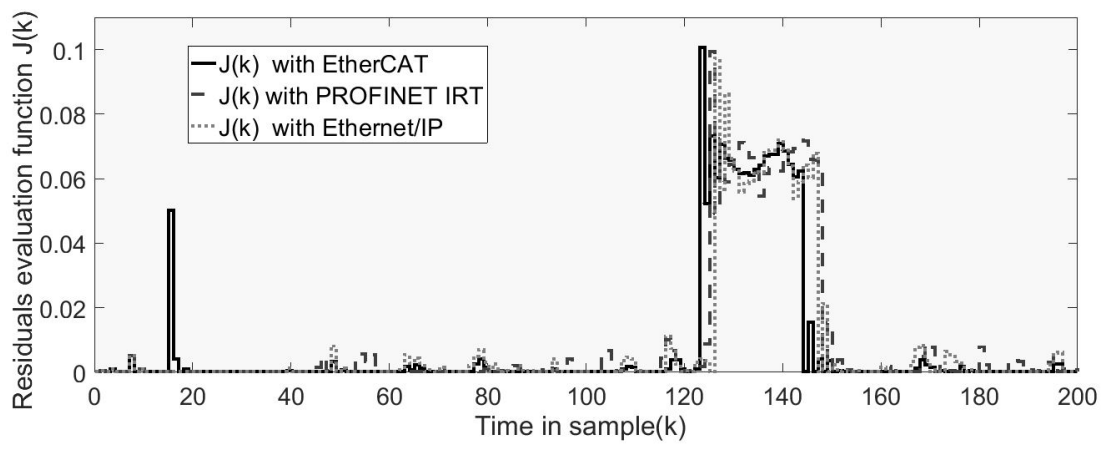

Figure 8. The residual evaluation function $\mathfrak{J}(k)$

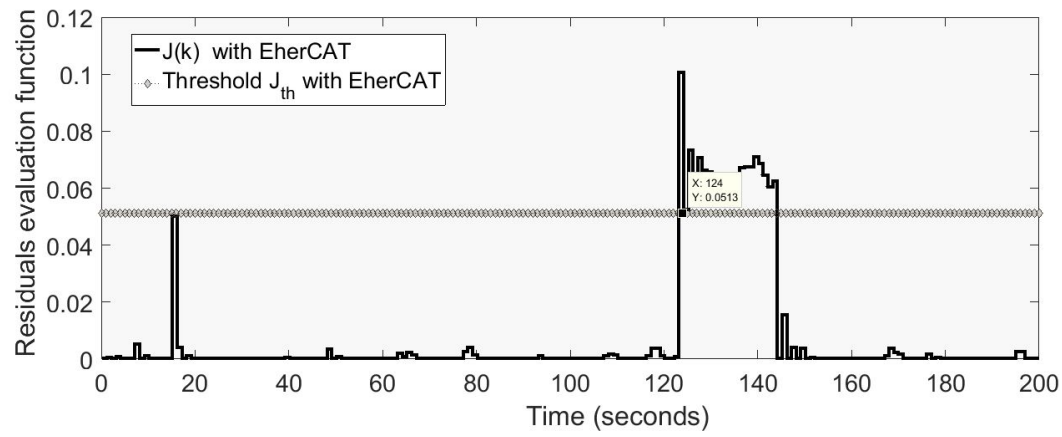

Figure 9. The residual evaluation function $\mathfrak{J}(k)$ with EtherCAT protocol 
According to (11), we can obtain the threshold of fault detection for EtherNet/IP protocol, PROFINET IRT protocol and EtherCAT protocol. The values of $\mathfrak{J}_{t h}$ and time steps that fault can be detected are reported in Table 2. We can notice that when fault occurs at steps 122, the occurrence of fault is detected at the steps 126 with EtherNet/IP protocol which corresponds to a delay of detection of steps 4 Figure 11.

According to Table 2, thecarriersensemultipleaccess/collisiondetection EtherCAT protocol will be adapted better to improve the performances of faults detection module in networked control hydroelectric power plant.

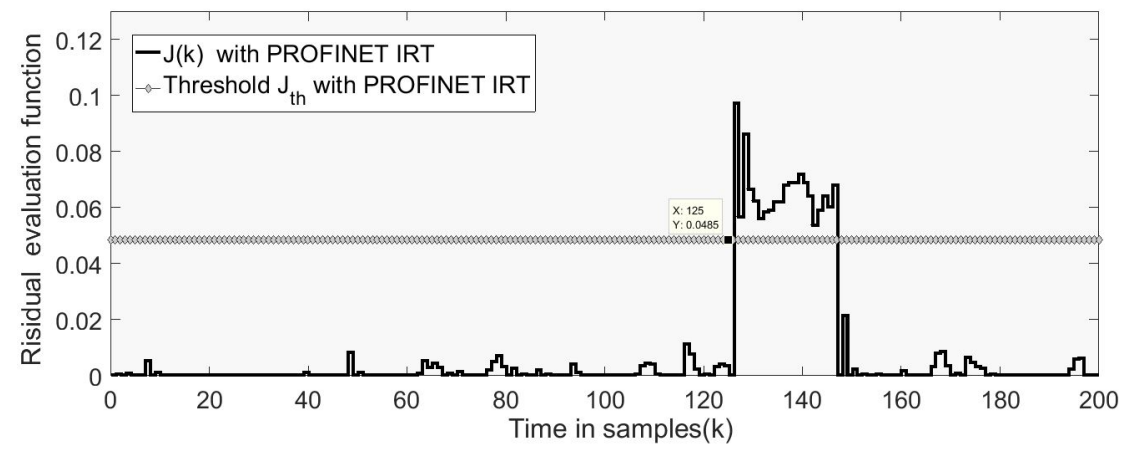

Figure 10. The residual evaluation function $\mathfrak{J}(k)$ with PROFINET IRT protocol

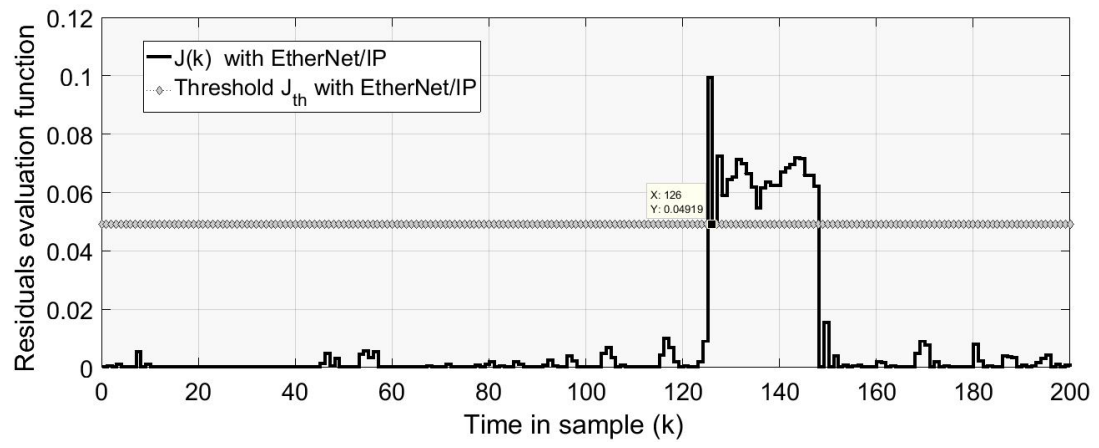

Figure 11. The residual evaluation function $\mathfrak{J}(k)$ with EtherNet/IP protocol

Table 2. Threshold $\mathfrak{J}_{t h}$ and time steps for different protocols

\begin{tabular}{lcr}
\hline Protocols & Threshold $\left(\mathfrak{J}_{t h}\right)$ & Time detection \\
\hline EtherNet/IP & 0.04919 & steps 126 \\
PROFINET IRT & 0.0485 & steps 125 \\
EtherCAT & 0.0524 & steps 124 \\
\hline
\end{tabular}

\section{CONCLUSION}

In this paper, an adaptive sliding mode observer-based fault detection for a class of NCSs subject to random communication delays, stochastic packet losses, access constraints and bounded disturbances has been established. A Lyapunov stability theory based on stochastic function is presented to analysis the asymptotical stability of the fault detection systems. The dynamic hydroelectric power plant model is used to verify the effectiveness of the proposed method based on TrueTime and Matlab/ Simulink, corroborated our predictions that a ethernet for control automation technology (EtherCAT) protocol would be more appropriate to reduce the false alarm rate and increasing the efficiency of the remote control hydroelectric power plant. Furthermore, the proposed results can be extended to the FD of NCSs with random communication delays, stochastic packet losses, access constraints quantization effects and possibly time-varying uncertainties. 


\section{REFERENCES}

[1] M. Yang, C. Peng, G. Li, "Event-triggered $H_{\infty}$ control for active semi-vehicle suspension system with communication constraints," Information Sciences, vol. 486, pp. 101-113, 2019, doi: 10.1016/j.ins.2019.02.047.

[2] W. P. M. H. Heemels, A. R. Teel, N. Van-de-Wouw, D. Nesic, "Networked Control Systems With Communication Constraints: Tradeoffs Between Transmission Intervals, Delays and Performance," IEEE Transactions on Automatic Control, vol. 55, no. 8, pp. 1781-1796, 2010, doi: 10.1109/TAC.2010.2042352.

[3] F. Khairandish, M. Akramian and M. Ataei, "Stability analysis and state feedback controller design for networked control systems with time varying delays," Modares Mechanical Engineering, vol. 17, no.5, pp: 77-85, 2017.

[4] N. Nafir , Z. Ahmida, K. Guelton, F. Bourahala and M. Rouamel, "Improved robust $H_{\infty}$ stability analysis and stabilization of uncertain and disturbed networked control systems with network-induced delay and packets dropout," International Journal of Systems Science in press, pp. 1-18, 2021, doi: 10.1080/00207721.2021.1931547.

[5] M. Rouamel, S. Gherbi and F. Bourahala, "Robust stability and stabilization of networked control systems with stochastic time-varying network-induced delays," Transactions of the Institute of Measurement and Control, vol. 42, no. 10, pp. 1782-1796, 2020, doi: 10.1177/0142331219895931.

[6] R. Pena-Alzola, M. Sztykiel, C. E. Jones , P. J. Norman, G. Moore, J. Pou and G. M. Burt , "First-fault detection in dc distribution with it grounding based on sliding discrete fourier-transform," IEEE Transactions on Power Electronics, vol. 36, no. 4 , pp. 3649-3654, 2020, doi: 10.1109/TPEL.2020.3026985.

[7] H. Zhou, Z. Liu and X. Yang, "Motor torque fault diagnosis for four wheel independent motordrive vehicle based on unscented kalman filter," IEEE Transactions on Vehicular Technology, vol. 67, no. 3, pp. 1969-1976, March 2018, doi: 10.1109/TVT.2017.2751750.

[8] W. Li, Y. Yan and J. Bao , "Dissipativity-based distributed fault diagnosis for plantwide chemical processes," Journal of Process Control, vol. 96, pp. 37-48, 2020, doi: 10.1016/j.jprocont.2020.10.007.

[9] L. Li, H. Luo, S. X. Ding, Y. Yang and K. Peng, "Performance-based fault detection and fault tolerant control for automatic control systems," Automatica, vol. 99, pp. 308-316, 2019, doi: 10.1016/j.automatica.2018.10.047.

[10] X. Liu, X. Su, P. Shi, S. K. Nguang and C. Shen , "Fault detection filtering for nonlinear switched systems via event-triggered communication approach," Automatica, vol. 101, pp. 365-376, 2019, doi: 10.1016/j.automatica.2018.12.006.

[11] Y. Pan and G. Yang, "Event-Triggered Fault Detection Filter Design for Nonlinear Networked Systems," in IEEE Transactions on Systems, Man, and Cybernetics: Systems, vol. 48, no. 11, pp. 1851-1862, Nov. 2018, doi: 10.1109/TSMC.2017.2719629.

[12] X. Chu and M. Li, " $H_{\infty}$ non-fragile observer-based dynamic event-triggered sliding mode control for nonlinear networked systems with sensor saturation and dead-zone input," ISA Transactions, vol. 94, pp. 93-107, 2019, doi: 0.1016/j.isatra.2019.04.018.

[13] Y. Wang, H. Ye, S.X. Ding, G. Wang, D. Zhou, "Residual generation and evaluation of networked control systems subject to random packet dropout," Automatica, vol. 5, no. 10, pp. 2427-2434 2009, doi: 10.1016/j.automatica.2009.06.031.

[14] P. Zhang, S. X. Ding, P. M. Frank and M. Sader, "Fault detection of networked control systems with missing measurements," 2004 5th Asian Control Conference (IEEE Cat. No.04EX904), pp. 1258-1263 vol. 2, 2004.

[15] J. Zhou and D. Zhang, "H-Infinity Fault Detection for Delta Operator Systems With Random TwoChannels Packet Losses and Limited Communication," in IEEE Access, vol. 7, pp. 94448-94459, 2019 , doi: 10.1109/ACCESS.2019.2928306.

[16] Y. Zhang, Z. Wang, L. Zou, and Z. Liu , "Fault detection filter design for networked multi-rate systems with fading measurements and randomly occurring faults," IET Control Theory Appl, vol. 10, n0. 5, pp. 573-581, 2016, doi: 10.1049/iet-cta.2015.0582.

[17] S. Chen, "Sliding mode observer design for discrete nonlinear time-delay systems with stochastic communication protocol," International Journal of Control, Automation and Systems, vol. 17, no.7, pp. 16661676, 2019. doi: 10.1007/s12555-018-0727-0.

[18] Y. M. Zhang and J. Jiang , "Bibliographical review on reconfigurable fault tolerant control systems," IFAC Proceedings Volumes, vol. 36, no. 5, pp. 257-268. 2003, doi: 10.1016/S1474-6670(17)36503-5. 
[19] J. Rohit, M. Abhinav. and S. Vinay, "Performance Evaluation of Fault Tolerance Protocols in MANET," International Journal of Computer Applications, vol. 61, no. 2, pp: 29-33, doi: 2013, 10.5120/9902-4490.

[20] W. Xuepei, X. Lihua, "Performance evaluation of industrial Ethernet protocols for networked control application," Control Engineering Practice, vol. 84, pp: 208-217, 2019. doi: 10.1016/j.conengprac.2018.11.022.

[21] A. H. Samba, A. T. Yeremou and L. Nneme Nneme, "Impacts of Packet Losses and Delay in a Networked Control Hydroelectric Power Plants," International Research Journal of Engineering and Technology , vol. 6, no. 2, pp. 1375-1384, 2019.

[22] A. H. Samba, A. Y. Tamtsia , I. J. H. Som and L. N. Nneme, "Networked iterative learning fault Diagnosis algorithm for systems with sensor random packet losses, time-varying delays, limited communication and actuator failure : Application to the hydroturbine Governing System," Wseas Transactions on Systems and Control, vol. 16, n0. 20, pp. 244-252, 2021, doi: 10.37394/23203.2021.16.20.

[23] L. Zhang and D. Hristu-Varsakelis, "Communication and control co-design for networked control systems," Automatica, vol. 42, no.6, pp. 953-958, 2006, doi: 10.1016/j.automatica.2006.01.022.

[24] E. E. Yaz, "Linear Matrix Inequalities In System And Control Theory," in Proceedings of the IEEE, vol. 86, no. 12, pp. 2473-2474, Dec. 1998, doi: 10.1109/JPROC.1998.735454.

[25] X. Y. Li and S. L. Sun, " $H_{\infty}$ control for networked systems with random delays and packet dropouts", Int. J. Control, Autom. Syst, vol. 10, n0. 5, pp. 1023-1031, 2012, doi: 10.1007/s12555-012-0520-4.

\section{BIOGRAPHIES OF AUTHORS}

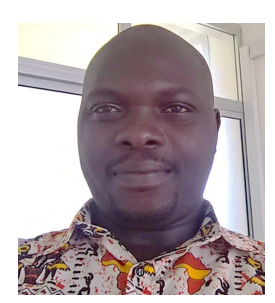

Samba Aimé Hervé received B.Sc. degree in Electronics, Electrical Engineering and Automation from Ngaoundere University, Cameroon in 2013 and the M.Sc. degree in Electronic and Instrumentation from Douala University, Cameroon, in 2016. His current work toward the Ph.D. degree in Automation and Industrial computer science at National Advanced School of Engineering, University of Douala, Cameroon. His research interests include iterative learning control, intelligent mechatronic systems, computational intelligence, fault detection and isolation (FDI), fault-tolerant control, networked control systems and computer distribute control systems. He has 7 publications in reputed journals. He serves as a reviewer invited of reputed journals such as IEEE Access, International Journal of Dynamics and Control (Springer nature) and Transaction of the Institute of Measurement and Control.

E-mail:aimehervesamba@yahoo.fr

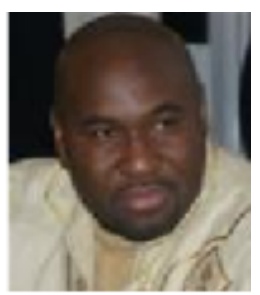

Yeremou Tamtsia Aurelien received the Master Degree in process engineering and the Ph.D. degree in vision and robotic from agro- food processing engineering school (ENSAI - Cameroon) and Blaise Pascal university Clermont- Ferrand (France) respectively. Now he is lecturer at National Advanced School of Engineering, University of Douala, Cameroon. His current researches lie on Robot Control, robust control, industrial instrumentation and regulations technologies of both deterministic and stochastic dynamic processes.

E-mail:ayeremou@yahoo.fr

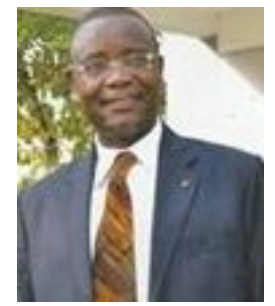

Nneme Nneme Leandre received the Master Degree in electrical and computer science engineering and the Ph.D. degree in Systems and Automation from Ecole Polytechnique of Montreal (Canada) in 1993 and 1998 respectively. From 1998, he held an academic position in the Advanced Teacher is Training College for Technical Education (ATTCTE) of Douala (Cameroon), as professor and Director of the ATTCTE. His current researches lie on Robot Control, industrial instrumentation technologies and intelligent control of both deterministic and stochastic dynamic processes.

E-mail: leandren@gmail.com 